\title{
The hadronic contribution to the running of the electromagnetic coupling and the electroweak mixing angle
}

\author{
Marco Cè, ${ }^{* a b \dagger}$ Teseo San José, ${ }^{* a b c}$ Antoine Gérardin, ${ }^{d}$ Harvey B. Meyer, ${ }^{a b c}$ Kohtaroh \\ Miura, ${ }^{a b e}$ Konstantin Ottnad, ${ }^{b c}$ Andreas Risch, ${ }^{b c}$ Jonas Wilhelm, ${ }^{b c}$ Hartmut Wittig ${ }^{a b c}$ \\ ${ }^{a}$ Helmholtz-Institut Mainz, Johannes Gutenberg-Universität Mainz, Germany \\ ${ }^{b}$ PRISMA ${ }^{+}$Cluster of Excellence, Johannes Gutenberg-Universität Mainz, Germany \\ ${ }^{c}$ Institut für Kernphysik, Johannes Gutenberg-Universität Mainz, Germany \\ ${ }^{d}$ John von Neumann-Institut für Computing (NIC), DESY Zeuthen, Germany \\ ${ }^{e}$ Kobayashi-Maskawa Institute for the Origin of Particles and the Universe, Nagoya University, \\ Japan \\ E-mail: marco.ce@uni-mainz.de, msanjosp@uni-mainz.de
}

The electromagnetic coupling $\alpha$ and the electroweak mixing angle $\theta_{\mathrm{W}}$ are parameters of the Standard Model (SM) that enter precision SM tests and play a fundamental rôle in beyond SM physics searches. Their values are energy dependent, and non-perturbative hadronic contributions are the main source of uncertainty to the theoretical knowledge of the running with energy. We present a lattice study of the leading hadronic contribution to the running of $\alpha$ and $\sin ^{2} \theta_{\mathrm{W}}$. The former is related to the hadronic vacuum polarization (HVP) function of electromagnetic currents, and the latter to the HVP mixing of the electromagnetic current with the vector part of the weak neutral currents. We use the time-momentum representation (TMR) method to compute the HVP on the lattice, estimating both connected and disconnected contributions on $N_{\mathrm{f}}=2+1$ nonperturbatively $\mathscr{O}(a)$-improved Wilson fermions ensembles from the Coordinated Lattice Simulations (CLS) initiative. The use of different lattice spacings and quark masses allows us to reliably extrapolate the results to the physical point.

MITP/19-064

DESY 19-179

37th International Symposium on Lattice Field Theory - Lattice2019

16-22 June 2019

Wuhan, China

\footnotetext{
*Speakers.

${ }^{\dagger}$ Current affiliation: Theoretical Physics department, CERN, Geneva, Switzerland
} 


\section{The running of the electromagnetic coupling}

The predictions of the Standard Model (SM) of particle physics are today tested to a high degree of precision in experiments that span a vast range of energy scales, from atomic physics to high-energy colliders. The connection between these energy scales is encoded in the running with energy of the strength of interactions. For instance, the fine-structure constant $\alpha=$ $1 / 137.035999139(31)$ [1] is known from low-energy experiments to better than a part per billion. However, its effective value for physics around the $Z$ pole is $\hat{\alpha}^{(5)}\left(M_{Z}\right)=1 / 127.955(10)$ [1], a $7 \%$ larger value. In the on-shell scheme, the running of $\alpha$ at a given time-like momentum transfer $q^{2}$ is described by

$$
\alpha\left(q^{2}\right)=\frac{\alpha}{1-\Delta \alpha\left(q^{2}\right)},
$$

in terms of the $\Delta \alpha\left(q^{2}\right)$ function. While the lepton contribution to $\Delta \alpha\left(q^{2}\right)$ can be computed in perturbation theory, the estimate of the quark contribution at low energies requires non-perturbative calculations of hadronic physics. Conventionally, the hadronic contribution $\Delta \alpha_{\text {had }}\left(q^{2}\right)$ is related through the optical theorem to the $R$-ratio, i.e. the total cross-section $e^{+} e^{-} \rightarrow$ hadrons over $e^{+} e^{-} \rightarrow$ $\mu^{+} \mu^{-}$, which is estimated using a compilation of experimental data. This results in $\Delta \alpha_{\text {had }}^{(5)}\left(M_{Z}^{2}\right)=$ $0.02764(7)$ [1] constituting the main contribution to the uncertainty on $\alpha\left(M_{Z}^{2}\right)$. In an alternative approach [2], the Adler function $D\left(Q^{2}\right)$ at space-like $Q^{2}=-q^{2}$ is estimated from the same data and used to compute the running up to $Q^{2} \approx 2 \mathrm{GeV}^{2}$. Around this $Q^{2}$ and above, $D\left(Q^{2}\right)$ can be computed reliably in perturbative QCD. Crucially, $\Delta \alpha_{\text {had }}\left(-Q^{2}\right)$ at space-like momenta $Q^{2}>0$ is accessible to non-perturbative lattice techniques defined in Euclidean space-time [3, 4, 5]. It is given by

$$
\Delta \alpha_{\mathrm{had}}\left(-Q^{2}\right)=4 \pi \alpha \bar{\Pi}^{\gamma \gamma}\left(Q^{2}\right), \quad \bar{\Pi}^{\gamma \gamma}\left(Q^{2}\right)=\left[\Pi^{\gamma \gamma}\left(Q^{2}\right)-\Pi^{\gamma \gamma}(0)\right],
$$

where $\bar{\Pi}\left(Q^{2}\right)$ is the subtracted hadronic vacuum polarization (HVP) function ${ }^{1}$

$$
\left(Q_{\mu} Q_{v}-\delta_{\mu v} Q^{2}\right) \Pi^{\gamma \gamma}\left(Q^{2}\right)=\Pi_{\mu \nu}^{\gamma \gamma}\left(Q^{2}\right)=\int \mathrm{d}^{4} x \mathrm{e}^{\mathrm{i} Q \cdot x}\left\langle j_{\mu}^{\gamma}(x) j_{v}^{\gamma}(0)\right\rangle
$$

of the electromagnetic current

$$
j_{\mu}^{\gamma}=\frac{2}{3} \bar{u} \gamma_{\mu} u-\frac{1}{3} \bar{d} \gamma_{\mu} d-\frac{1}{3} \bar{s} \gamma_{\mu} s+\frac{2}{3} \bar{c} \gamma_{\mu} c .
$$

Lattice QCD can thus provide an estimate that does not depend on experimental $R$-ratio data and cross-check the phenomenological estimate. This is of great interest in the context of global SM fits, where $\Delta \alpha_{\text {had }}\left(M_{Z}^{2}\right)$ is an input. Indeed, the best-fit result for the Higgs mass excluding kinematic constraints is $M_{H}=90_{-16}^{+17} \mathrm{GeV}, 1.9 \sigma$ below the measured value, and a shift of $\pm 10^{-4}$ in $\Delta \alpha_{\text {had }}^{(3)}\left(4 \mathrm{GeV}^{2}\right)$ corresponds to a shift of $\mp 4.5 \mathrm{GeV}$ in $M_{H}$ [1]. This is connected to the tension between the SM and experimental determinations of the anomalous magnetic moment of the muon $(g-2)_{\mu}$ (see Ref. [6] for a review), because a solution of the $(g-2)_{\mu}$ puzzle that involves an increase of the SM estimate of the leading hadronic contribution $a_{\mu}^{\mathrm{HLO}}$ has to avoid a correlated increase of $\Delta \alpha_{\text {had }}$ [7].

\footnotetext{
${ }^{1}$ In this work we denote with $\bar{\Pi}\left(Q^{2}\right)$ with $Q^{2}>0$ the HVP function at space-like momenta.
} 
A second connection to $(g-2)_{\mu}$ comes from the high-precision measurement of $\alpha\left(-Q^{2}\right)$ at space-like $Q^{2}$ in $t$-channel scattering proposed by the MUonE collaboration [8, 9]. Isolating the hadronic contribution $\Delta \alpha_{\text {had }}\left(-Q^{2}\right)$, an independent determination of $a_{\mu}^{\mathrm{HLO}}$ is obtained from [10]

$$
a_{\mu}^{\mathrm{HLO}}=\frac{\alpha}{\pi} \int_{0}^{1} \mathrm{~d} x(1-x) \Delta \alpha_{\mathrm{had}}(t(x)), \quad t(x)=\frac{x^{2} m_{\mu}^{2}}{x-1} \leq 0 .
$$

The proposed experiment is limited to $x<0.932$, corresponding to $Q^{2} \lesssim 0.14 \mathrm{GeV}^{2}$, that leaves out $13 \%$ of the $a_{\mu}^{\mathrm{HLO}}$ integral. Lattice data can complement the experiment for $Q^{2} \gtrsim 0.14 \mathrm{GeV}^{2}$ [11].

\section{The running of the electroweak mixing angle}

As a second quantity, we consider the electroweak mixing angle or Weinberg angle $\theta_{\mathrm{W}}$, that is, the parameter of the Standard Model of particle physics that parametrizes the mixing between electromagnetic and weak interactions

$$
\sin ^{2} \theta_{\mathrm{W}}=\frac{g^{\prime 2}}{g^{2}+g^{\prime 2}}, \quad e=g \sin \theta_{\mathrm{W}}=g^{\prime} \cos \theta_{\mathrm{W}}
$$

where $g$ and $g^{\prime}$ are the $\mathrm{SU}(2)_{L}$ and $\mathrm{U}(1)_{Y}$ couplings, respectively. Beyond tree level, as the couplings themselves, its precise value is scheme and energy dependent. In a given scheme, the mixing angle is measured to sub-permille precision at energies around $M_{Z}$, e.g. on-shell $\sin ^{2} \theta_{\mathrm{W}}=1-M_{W}^{2} / M_{Z}^{2}=$ $0.22343(7)$ or in the $\overline{\mathrm{MS}}$-scheme $\sin ^{2} \hat{\theta}_{\mathrm{W}}\left(M_{Z}\right)=0.23122$ (7) [1]. Conversely, the mixing angle in the Thomson limit, $\left|q^{2}\right| \ll m_{e}^{2}$, can be defined in a scheme-independent way [12,13]. Its experimental value is less well known, but upcoming experiments target up to a $0.15 \%$ precision at a momentum transfer of $4.5 \times 10^{-3} \mathrm{GeV}^{2}[14,15]$. A more precise value, $\sin ^{2} \hat{\theta}_{\mathrm{W}}(0)=0.23868(5)(2)$ [16], is obtained computing the running in the $\overline{\mathrm{MS}}$-scheme from $M_{Z}$ to low energies. The first error is the uncertainty on the $Z$-pole value, while the second error is the total theoretical uncertainty on the running and it is dominated by the non-perturbative hadronic contribution.

The energy dependence of $\sin ^{2} \theta_{\mathrm{W}}$ in the on-shell scheme can be written as

$$
\sin ^{2} \theta_{\mathrm{W}}\left(q^{2}\right)=\sin ^{2} \theta_{\mathrm{W}}\left[1+\Delta \sin ^{2} \theta_{\mathrm{W}}\left(q^{2}\right)\right]
$$

where $\sin ^{2} \theta_{\mathrm{W}}$ is the value in the low-energy limit. Similarly to Eq (1.2), the leading hadronic contribution to the running at space-like $Q^{2}=-q^{2}$ is given by [17, 2]

$$
\Delta_{\text {had }} \sin ^{2} \theta_{\mathrm{W}}\left(-Q^{2}\right)=-\frac{e^{2}}{\sin ^{2} \theta_{\mathrm{W}}} \bar{\Pi}^{Z \gamma}\left(Q^{2}\right),
$$

where $\bar{\Pi}^{Z \gamma}\left(Q^{2}\right)$ is HVP mixing of the electromagnetic current $j_{\mu}^{\gamma}$ and the vector part of the neutral weak current $j_{\mu}^{Z}$

$$
\left.j_{\mu}^{Z}\right|_{\text {vector }}=\left.j_{\mu}^{T_{3}}\right|_{\text {vector }}-\sin ^{2} \theta_{\mathrm{W}} j_{\mu}^{\gamma},\left.\quad j_{\mu}^{T_{3}}\right|_{\text {vector }}=\frac{1}{4} \bar{u} \gamma_{\mu} u-\frac{1}{4} \bar{d} \gamma_{\mu} d-\frac{1}{4} \bar{s} \gamma_{\mu} s+\frac{1}{4} \bar{c} \gamma_{\mu} c
$$

As in the electromagnetic case, $\bar{\Pi}^{Z \gamma}\left(Q^{2}\right)$ is directly accessible to lattice computations $[3,4,18,19]$. 

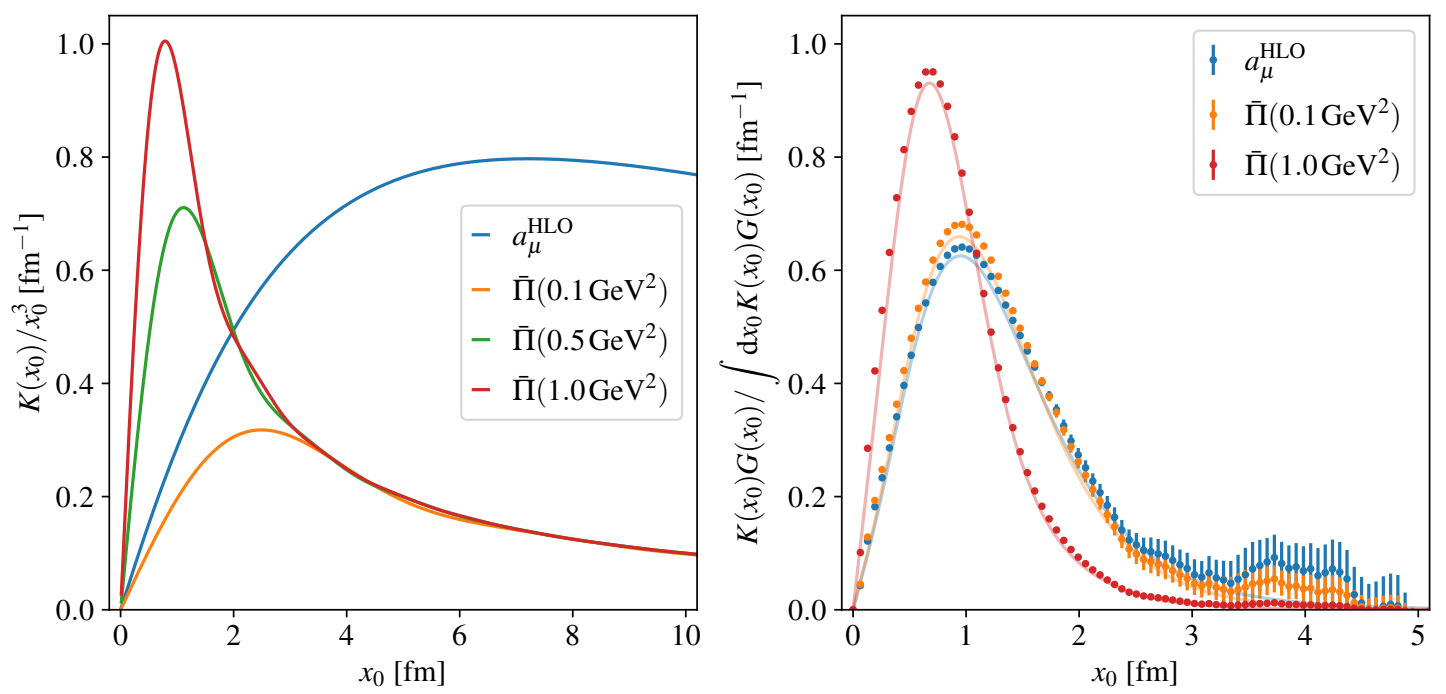

Figure 1: Left: the kernel $K\left(x_{0}, Q^{2}\right)$ of the TMR integral in Eq. (3.1) divided by $x_{0}^{3}$ for different values of $Q^{2}$, compared to the kernel for $a_{\mu}^{\mathrm{HLO}}[20,21]$ (blue line), as a function of time $x_{0}$. Right: contribution of $G\left(x_{0}\right) K\left(x_{0}, Q^{2}\right)$ to the TMR integral normalized to the value of the integral, comparing different kernels $K\left(x_{0}\right)$. The light coloured lines are drawn using a model for the Euclidean-time correlator $G\left(x_{0}\right)$ [20], that is also used for the integral, while the data points with error bars are obtained using actual lattice correlator data at the physical pion mass.

\section{The TMR method}

The computation of $\bar{\Pi}^{\gamma \gamma}$ and $\bar{\Pi}^{Z \gamma}$ as functions of $Q^{2}$ is similar to that of $a_{\mu}^{\mathrm{HLO}}$, the leading-order HVP contribution to $(g-2)_{\mu}$, and, as in that case, different methods are available, such as the four-momentum hybrid method, the time moments, or the time-momentum representation (TMR) method [20, 22]. In this study, we employ the TMR method to compute the subtracted HVP function

$$
\bar{\Pi}\left(Q^{2}\right)=\int_{0}^{\infty} \mathrm{d} x_{0} G\left(x_{0}\right)\left[x_{0}^{2}-\frac{4}{Q^{2}} \sin ^{2}\left(\frac{Q x_{0}}{2}\right)\right], \quad G\left(x_{0}\right)=-\frac{1}{3} \int \mathrm{d}^{3} x \sum_{k=1}^{3}\left\langle j_{k}(x) j_{k}(0)\right\rangle,
$$

where we have to integrate over Euclidean time the product of the zero-momentum-projected correlator, $G^{\gamma \gamma}\left(x_{0}\right)$ or $G^{Z \gamma}\left(x_{0}\right)$, times a $Q^{2}$-dependent kernel $K\left(x_{0}, Q^{2}\right)=x_{0}^{2}-\left(4 / Q^{2}\right) \sin ^{2}\left(Q x_{0} / 2\right)$. This allows us, in principle, to input any value of $Q^{2}$ in the kernel. The properties of the kernel significantly influence the systematics of the integral: On the one hand, a shorter-range kernel puts a larger weight on the correlator at short times. Since the correlator on the lattice is sampled at a spacing $a$, one needs $Q^{2} \ll(\pi / a)^{2}$ in order to avoid large cut-off effects. On the other hand, a longer-range kernel weights relatively more the long-time behaviour of the correlator, which is noisier and susceptible to finite-volume effects. Different kernels are compared in the left plot of Figure 1, including the one used to compute $a_{\mu}^{\mathrm{HLO}}$, Eq. (84) from Ref. [20]. In the right plot we show the corresponding relative contribution to the integral against the time variable. The HVP function $\bar{\Pi}$ at $Q^{2}=0.1 \mathrm{GeV}^{2}$ receives the larger contribution around $1 \mathrm{fm}$, as $a_{\mu}^{\mathrm{HLO}}$ does, but at long times the contribution to the former is smaller. When lattice data at physical pion masses is used for $G\left(x_{0}\right)$, 
Table 1: List of ensembles from the CLS initiative employed, with approximate lattice spacings, sizes and pion and kaon masses. A* denotes the ensembles for which the disconnected contribution is available. All ensembles have open boundary conditions in time, except those denoted with ${ }^{\S}$ that have periodic boundary conditions in time. Values of $t_{0}^{\mathrm{sym}}$ and $a$ are from Ref. [25].

\begin{tabular}{lrccccccc}
\hline & $T / a$ & $L / a$ & $t_{0}^{\mathrm{sym}} / a^{2}$ & $a[\mathrm{fm}]$ & $L[\mathrm{fm}]$ & $M_{\pi}, M_{K}[\mathrm{MeV}]$ & $M_{\pi} L$ \\
\hline $\mathrm{H} 101$ & 96 & 32 & 2.860 & 0.086 & 2.8 & \multicolumn{2}{c}{415} & 5.8 \\
$\mathrm{H} 102$ & 96 & 32 & & & 2.8 & 355 & 440 & 5.0 \\
$\mathrm{H} 105^{*}$ & 96 & 32 & & & 2.8 & 280 & 460 & 3.9 \\
$\mathrm{~N} 101$ & 128 & 48 & & & 4.1 & 280 & 460 & 5.8 \\
$\mathrm{C} 101^{*}$ & 96 & 48 & & & 4.1 & 220 & 470 & 4.6 \\
\hline B450 & 64 & 32 & 3.659 & 0.076 & 2.4 & & 415 & 5.1 \\
S400 & 128 & 32 & & & 2.4 & 350 & 440 & 4.3 \\
N401* & 128 & 48 & & & 3.7 & 285 & 460 & 5.3 \\
\hline H200 & 96 & 32 & 5.164 & 0.064 & 2.1 & & 420 & 4.4 \\
N202 & 128 & 48 & & & 3.1 & & 410 & 6.4 \\
N203* & 128 & 48 & & & 3.1 & 345 & 440 & 5.4 \\
N200* & 128 & 48 & & & 3.1 & 285 & 465 & 4.4 \\
D200* & 128 & 64 & & & 4.1 & 200 & 480 & 4.2 \\
E250*8 & 192 & 96 & & & 6.2 & 130 & 490 & 4.1 \\
\hline N300 & 128 & 48 & 8.595 & 0.050 & 2.4 & & 420 & 5.1 \\
N302* & 128 & 48 & & & 2.4 & 345 & 460 & 4.2 \\
J303 & 192 & 64 & & & 3.2 & 260 & 475 & 4.2 \\
\hline
\end{tabular}

this results in a smaller statistical error on $\bar{\Pi}\left(0.1 \mathrm{GeV}^{2}\right)$ than on $a_{\mu}^{\mathrm{HLO}}$. In the case of $\bar{\Pi}\left(1 \mathrm{GeV}^{2}\right)$, the correlator tail has a negligible contribution to the statistical error.

\section{Lattice setup}

We perform the computation on the $N_{\mathrm{f}}=2+1$ set of ensembles from the Coordinated Lattice Simulations (CLS) initiative [23], with tree-level Lüscher-Weisz gauge action and non-perturbatively $\mathscr{O}(a)$-improved Wilson fermions. The list of ensembles employed in this work is in Table 1 . We use four lattice spacings, $u$ and $d$ quark masses are degenerate, thus we have exact isospin symmetry, and the pseudoscalar meson masses span from $M_{\pi}=M_{K} \approx 415 \mathrm{MeV}$ at the SU(3)-symmetric point to the physical ones along a trajectory on which the sum of the bare $u, d$ and $s$ quark masses is kept constant. We set the scale using $\left(8 t_{0}^{\text {phys }}\right)^{1 / 2}=0.415(4)(2)$ fm [24, 25].

\subsection{Flavour decomposition and renormalization}

The correlators are computed on the ensembles in Table 1 as described in Ref. [26], to which we refer to for the unexplained notation. At the sink, we employ both the local and conserved 
discretizations of the vector current

$$
\begin{gathered}
j_{\mu}^{1}(x)=\bar{q}(x) \gamma_{\mu} q(x), \\
j_{\mu}^{\mathrm{c}}(x)=\frac{1}{2}\left[\bar{q}(x+a \hat{\mu})\left(1+\gamma_{\mu}\right) U_{\mu}^{\dagger}(x) q(x)-\bar{q}(x)\left(1-\gamma_{\mu}\right) U_{\mu}(x) q(x+a \hat{\mu})\right],
\end{gathered}
$$

while only the local current is used at the source. The currents are non-perturbatively $\mathscr{O}(a)$-improved and renormalized. To this purpose, we introduce a flavour $\mathrm{SU}(3)$ decomposition of the current. For the local discretization, we have [27]

$$
\begin{gathered}
j_{\mu, R}^{3,1}=Z_{V}\left(1+3 \bar{b}_{V} a m_{q}^{\mathrm{av}}+b_{V} a m_{q, \ell}\right) j_{\mu}^{3, \mathrm{il}}, \\
\left(\begin{array}{c}
j_{\mu}^{8} \\
j_{\mu}^{0}
\end{array}\right)_{R}^{1}=Z_{V}\left(\begin{array}{cc}
1+3 \bar{b}_{V} a m_{q}^{\mathrm{av}}+b_{V} \frac{a\left(m_{q, \ell}+2 m_{q, s}\right)}{3} & \left(\frac{b_{V}}{3}+f_{V}\right) \frac{2 a\left(m_{q, \ell}-m_{q, s}\right)}{\sqrt{3}} \\
r_{V} d_{V} \frac{a\left(m_{q, \ell}-m_{q, s}\right)}{\sqrt{3}} & r_{V}+r_{V}\left(3 \bar{d}_{V}+d_{V}\right) a m_{q}^{\mathrm{av}}
\end{array}\right)\left(\begin{array}{c}
j_{\mu}^{8} \\
j_{\mu}^{0}
\end{array}\right)^{\mathrm{il}},
\end{gathered}
$$

where the improved non-singlet and singlet local currents are

$$
j_{\mu}^{a, i 1}=j_{\mu}^{a, 1}+a c_{V}^{1} \tilde{\partial}_{v} T_{v \mu}^{a}, \quad j_{\mu}^{0, i 1}=j_{\mu}^{0,1}+a \bar{c}_{V}^{1} \tilde{\partial}_{\nu} T_{\nu \mu}^{0},
$$

and the breaking of flavour $\mathrm{SU}(3)$ symmetry introduces a mixing between the singlet and non-singlet $I=0$ components. For the conserved discretization, no renormalization is needed

$$
j_{\mu, R}^{a, \mathrm{c}}=j_{\mu}^{a, \mathrm{c}}+a c_{V}^{\mathrm{c}} \tilde{\partial}_{\nu} T_{v \mu}^{a}, \quad j_{\mu, R}^{0, \mathrm{c}}=j_{\mu}^{0, \mathrm{c}}+a \bar{c}_{V}^{\mathrm{c}} \tilde{\partial}_{\nu} T_{\nu \mu}^{0} .
$$

We use the renormalization and improvement coefficients determined non-perturbatively in Ref. [28]. Since the coefficients to renormalize the singlet local current are unknown, we use only the conserved vector current for the singlet component. This implies that we can only put the $Z$ current at the sink, where the conserved discretization is available. Moreover, we set $f_{V}=0$ and $\bar{c}_{V}^{\mathrm{c}, 1}=c_{V}^{\mathrm{c}, 1}$, which is valid up to $\mathscr{O}\left(g_{0}^{5}\right)$ and introduces a negligible error.

The charm contribution is also computed in the quenched approximation, with the charm quark mass tuned using the experimental $D_{s}$ meson mass and the local current renormalized computing the mass-dependent $Z_{V}^{c}$ [26]. Including the latter contribution, the $\gamma \gamma$ and $Z \gamma$ bare correlators are

$$
\begin{gathered}
G_{\mu v}^{\gamma \gamma}(x)=G_{\mu v}^{33}(x)+\frac{1}{3} G_{\mu v}^{88}(x)+\frac{4}{9} C_{\mu v}^{c, c}(x), \\
G_{\mu v}^{Z \gamma}(x)=\left(\frac{1}{2}-\sin ^{2} \theta_{\mathrm{W}}\right) G_{\mu \nu}^{\gamma \gamma}(x)-\frac{1}{6 \sqrt{3}} G_{\mu \nu}^{08}(x)-\frac{1}{18} C_{\mu \nu}^{c, c}(x),
\end{gathered}
$$

where the flavour $\mathrm{SU}(3)$ contributions are defined as ${ }^{2}$

$$
\begin{gathered}
G_{\mu \nu}^{33}(x)=\frac{1}{2} C_{\mu v}^{\ell, \ell}(x), \\
G_{\mu \nu}^{88}(x)=\frac{1}{6}\left[C_{\mu \nu}^{\ell, \ell}(x)+2 C_{\mu \nu}^{s, s}(x)+2 D_{\mu \nu}^{\ell-s, \ell-s}(x)\right], \\
G_{\mu \nu}^{08}(x)=\frac{1}{2 \sqrt{3}}\left[C_{\mu \nu}^{\ell, \ell}(x)-C_{\mu \nu}^{s, s}(x)+D_{\mu \nu}^{2 \ell+s, \ell-s}(x)\right],
\end{gathered}
$$

and the connected and disconnected Wick's contractions are

$$
\begin{gathered}
C_{\mu \nu}^{f_{1}, f_{2}}(x)=-\left\langle\operatorname{Tr}\left\{D_{f_{1}}^{-1}(x, 0) \gamma_{\mu} D_{f_{2}}^{-1}(0, x) \gamma_{v}\right\}\right\rangle, \\
D_{\mu \nu}^{f_{1}, f_{2}}(x)=\left\langle\operatorname{Tr}\left\{D_{f_{1}}^{-1}(x, x) \gamma_{\mu}\right\} \operatorname{Tr}\left\{D_{f_{2}}^{-1}(0,0) \gamma_{v}\right\}\right\rangle .
\end{gathered}
$$

\footnotetext{
${ }^{2}$ In the usual lattice notation, $G_{\mathrm{conn}}^{\ell}=2 G^{33}$ and $G_{\mathrm{conn}}^{s}=3 G_{\mathrm{conn}}^{88}-G^{33}$. Moreover, $G_{\mathrm{conn}}^{08}=\sqrt{3}\left(G^{33}-G_{\mathrm{conn}}^{88}\right) / 2$.
} 


\subsection{Autocorrelation study}

Due to the update procedure of Monte Carlo simulations different configurations within one chain are not independent. In order to give a reliable estimate of the observable's uncertainty we need to take these autocorrelations into account. For this work we have used the $\Gamma$-method $[29,30]$ to estimate the autocorrelation time, $\tau_{\text {int }}$ and, from it, an appropriate bin size to obtain statistically independent samples. Since autocorrelations are observable-dependent we have computed $\tau_{\text {int }}$ of $\Delta \alpha_{\text {had }}$ for the light and strange flavours on each ensemble at different energies. Extra caution needs to be taken regarding the tail of the correlator, which shows the signal-to-noise ratio problem (see Section 4.4). To obtain a good estimate of $\tau_{\text {int }}$ we only take into account the correlator until a certain maximum value of $x_{0}$. Repeating the process for different time cuts, we know where the noise of the tail starts to dominate and the value of $\tau_{\text {int }}$. We choose a bin size $B=2 \tau_{\text {int }}$ for each ensemble to bin the correlator data and obtain a distribution of independent bootstrap samples to carry out the main analysis.

\subsection{Finite-size corrections}

In this work, we estimate finite-volume corrections using the same strategy described in Ref. [26]. Namely, we compute the difference between the infinite- and finite-volume $I=1$ correlator and apply it as a correction to the lattice data. We use two distinct models to compute the difference, depending on whether the correlator is considered at short or long times.

At long times, we use the $I=1$ correlator obtained from the time-like pion form factor $F_{\pi}(\omega)$. In infinite volume, that is

$$
G^{33}\left(x_{0}, \infty\right)=\int_{0}^{\infty} \mathrm{d} \omega \omega^{2} \rho\left(\omega^{2}\right) \mathrm{e}^{-\omega x_{0}}, \quad \rho\left(\omega^{2}\right)=\frac{1}{48 \pi^{2}}\left(1-\frac{4 M_{\pi}^{2}}{\omega^{2}}\right)^{\frac{3}{2}}\left|F_{\pi}(\omega)\right|^{2},
$$

while the finite volume expression is computed from the Lüscher energies $\omega_{n}$ and the LellouchLüscher amplitudes,

$$
G^{33}\left(x_{0}, L\right)=\sum_{n}\left|A_{n}\right|^{2} \mathrm{e}^{-\omega_{n} x_{0}},
$$

extracted applying the Lellouch-Lüscher formalism [31, 32, 33]. In this work, we use the GounarisSakurai (GS) parametrization [34] of $F_{\pi}(\omega)$, that depends on two parameters, $m_{\rho}$ and $g_{\rho \pi \pi}$, which are obtained either by fitting the correlator at long times or by a spectroscopic analysis [26]. We emphasise that the model is used only to correct for the relatively small finite-volume effect of the correlator, while no modelling of the contribution from the correlator tail is assumed. In the future, we plan to further reduce the model-dependence employing, where available, a full lattice determination of $F_{\pi}(\omega)[35,36]$ instead of the GS parametrization.

The $F_{\pi}(\omega)$-based model provides a good spectral representation of the correlator up to the threepion threshold, thus we use it for the correlator correction at times $x_{0}>x_{0 i}$, with $x_{0 i}=\left(M_{\pi} L / 4\right)^{2} / M_{\pi}$. At smaller times $x_{0} \leq x_{0 i}$ we compute the difference between the infinite- and finite-volume correlator in scalar QED (i.e. NLO $\chi \mathrm{PT})[22,21]$

$$
G^{33}\left(x_{0}, \infty\right)-G^{33}\left(x_{0}, L\right)=\frac{1}{3}\left(\int \frac{\mathrm{d}^{3} \vec{k}}{(2 \pi)^{3}}-\frac{1}{L^{3}} \sum_{\vec{k}}\right) \frac{\vec{k}^{2}+M_{\pi}^{2}}{\vec{k}^{2}} \mathrm{e}^{-2 x_{0} \sqrt{\vec{k}^{2}+M_{\pi}^{2}}} .
$$

This model omits the pion self-interaction and it is known to only account for a fraction of the finitevolume correction to $\bar{\Pi}\left(Q^{2}\right)$ at $Q^{2}$ values of $\mathscr{O}\left(\mathrm{GeV}^{2}\right)$ [37]. However, comparing the correlator on 
two pairs of ensembles in Table 1 with the same parameters except for different physical volumes, we observe that the finite-volume correction at times $x_{0} \leq x_{0 i}$ is smaller than our uncertainties. Therefore, we are confident that this rather simple short-time description is sufficient at the current level of precision, while recent developments [38] show a promising path towards improving on it.

We can estimate the relative size of the finite-volume corrections. For example, on ensembles D200, N302 and J303, the ratio of the correction over the total $\Delta \alpha_{\text {had }}$ at $Q^{2}=1 \mathrm{GeV}^{2}$ amounts to more than $1 \%$. The contribution, although small in absolute terms, is already bigger than the $0.5 \%$ statistical uncertainty on these ensembles and therefore needs to be included.

\subsection{Signal-to-noise ratio}

Both the correlator and its variance can be expressed, using the spectral decomposition, as an infinite tower of exponentials, but with different decay rates and amplitudes. In general, this leads to an exponential deterioration of the signal with respect to the noise with Euclidean time, known as the signal-to-noise ratio problem. As shown in Section 3, this problem has a larger impact on the statistical error of $\bar{\Pi}\left(Q^{2}\right)$ at smaller $Q^{2}$. Although it affects all flavour components, it is more acute for the light correlator, whose contribution remains sizeable longer in time.

As described in Ref. [21], one possibility is to model the tail of the correlator with a single exponential, i.e.

$$
G\left(x_{0}\right)= \begin{cases}\text { data, } & x_{0}<x_{0}^{\text {cut }}, \\ A \mathrm{e}^{-m_{V} x_{0}}, & x_{0} \geq x_{0}^{\text {cut }},\end{cases}
$$

where $A$ and $m_{V}$ are obtained fitting the correlator. In our case, due to the high statistics available, $x_{0}^{\text {cut }}$ lies between $1.5 \mathrm{fm}$ and $2.5 \mathrm{fm}$, depending on the ensemble. However, there is no guarantee that a single state describes the tail of the correlator. This is particularly true for light ensembles in a large volume, where both the $\rho$ resonance and the tower of two- $\pi$ states are long range contributions. Therefore, this method has its limitations, and we choose not to use it. The results presented in the following sections are obtained using the correlator data until the last time slice available. As explained in Section 3, the contribution from the tail to $\bar{\Pi}\left(Q^{2}\right)$ is smaller than in the case of $a_{\mu}^{\mathrm{HLO}}$.

In the future, we are considering improving the statistical error on $\bar{\Pi}\left(Q^{2}\right)$ at small $Q^{2}$, especially on light-pion ensembles, substituting the correlator at long times with a spectral reconstruction, and controlling the systematics e.g. with the bounding method [39, 26]. In its simplest incarnation, this relies on the fact that $G\left(x_{0}\right)$ at $x_{0} \geq x_{0}^{\text {cut }}$ is bounded by

$$
0 \leq G\left(x_{0}^{\text {cut }}\right) \mathrm{e}^{-E_{\text {eff }}\left(x_{0}^{\text {cut }}\right)\left(x_{0}-x_{0}^{\text {cut }}\right)} \leq G\left(x_{0}\right) \leq G\left(x_{0}^{\text {cut }}\right) \mathrm{e}^{-E_{0}\left(x_{0}-x_{0}^{\text {cut }}\right)},
$$

where $E_{\text {eff }}\left(x_{0}\right)=-\mathrm{d} \log \left(G\left(x_{0}\right)\right) / \mathrm{d} x_{0}$ is the effective mass and $E_{0}$ the ground state energy, to obtain the $x_{0}^{\text {cut }}$ so both bounds yield the same result for the observable.

\section{Numerical results}

Figure 2 shows the running of different contributions to $\bar{\Pi}\left(Q^{2}\right)$, defined through the correlators in Eq. (4.5), as a function of $Q^{2}$ on three different lattices at the same lattice spacing with increasingly lighter pions. As one moves away from the SU(3)-symmetric point, the $\bar{\Pi}^{33}$ contribution increases while the $\bar{\Pi}_{\text {conn }}^{88}$ contribution decreases. The (quenched) charm contribution is also shown to be relatively independent on the pion mass and linearly increasing in the range of $Q^{2}$ values. The 


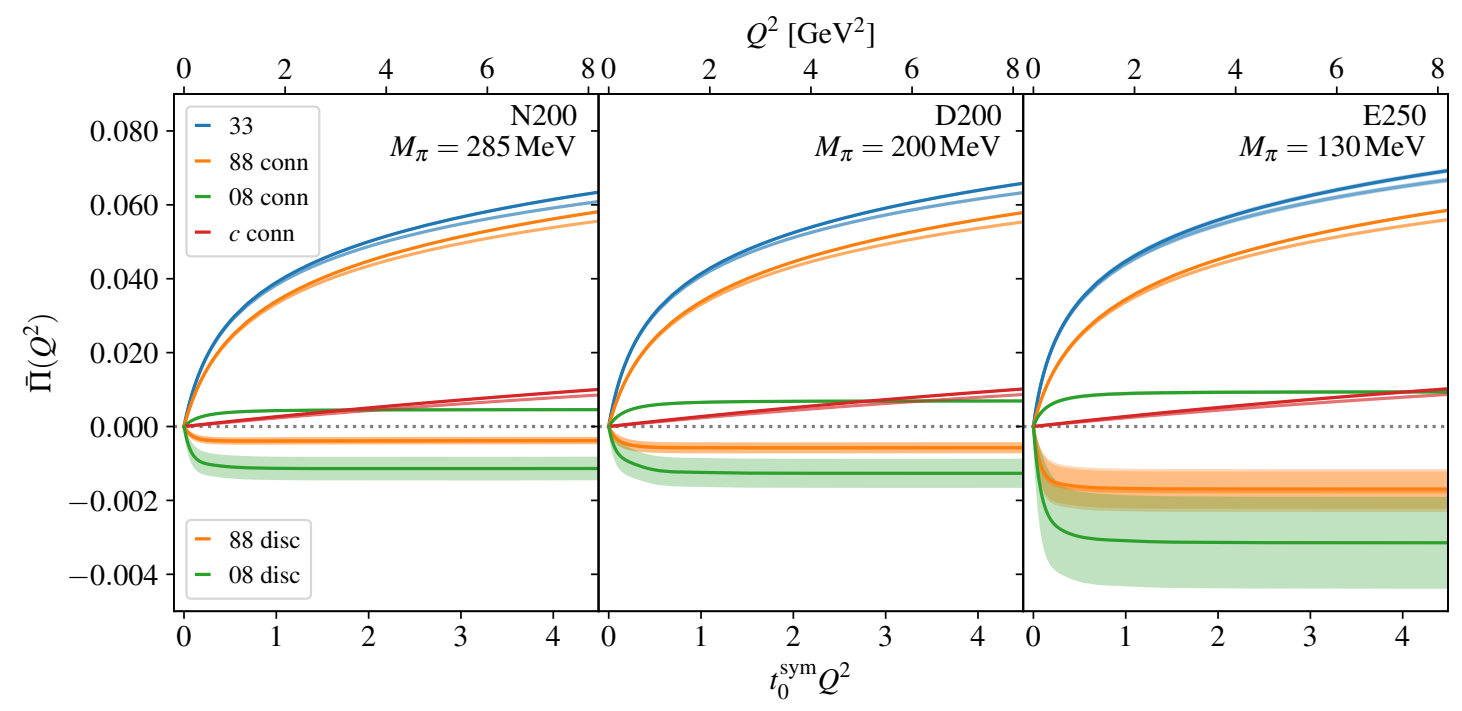

Figure 2: Running with energy $Q^{2}$ of different contributions to $\bar{\Pi}\left(Q^{2}\right)$ on three different ensembles at $a \approx 0.064 \mathrm{fm}$. The conserved, local discretization is shown and, when available, the local, local discretization in a lighter colour shade. The negative side of the vertical axis of the plot is inflated by a factor 10 with respect to the positive side.

negative disconnected contributions are also shown, enlarged by a factor 10 . They are obtained cutting the TMR integration at $x_{0}^{\text {cut }} \approx 2.5 \mathrm{fm}$, which results in a conservative statistical error. Nevertheless, Table 2 shows that the statistical error on the disconnected contribution is comparable to the connected one. The exception is the E250 ensemble, with only a small number of disconnected loop measurements being available at the current time. We will improve the disconnected contribution estimate by implementing the bounding method for the $I=0$ channel, in order to reduce the statistical error and correctly estimate the integration tail systematics. It is worth noting that $\bar{\Pi}_{\text {disc }}^{88}\left(Q^{2}\right)$ is constant for $Q^{2} \gtrsim 0.5 \mathrm{GeV}^{2}$, as predicted by perturbation theory.

\subsection{Extrapolation to the physical point}

Four different lattice spacings and several quark masses, including the physical ones, allow us to

Table 2: Estimate of connected and disconnected contributions to $10^{5} \cdot \bar{\Pi}\left(1 \mathrm{GeV}^{2}\right)$ for the conserved-local (c.1.) and, when available, local-local (1.1.) discretizations, on three different ensembles at $a \approx 0.064 \mathrm{fm}$.

\begin{tabular}{rcccccc}
\hline$\times 10^{-5}$ & $\bar{\Pi}^{33}$ & $\bar{\Pi}_{\text {conn }}^{88}$ & $\bar{\Pi}_{\text {conn }}^{08}$ & $\bar{\Pi}_{\text {conn }}^{c}$ & $\bar{\Pi}_{\text {disc }}^{88}$ & $\bar{\Pi}_{\text {disc }}^{08}$ \\
\hline $\mathrm{N} 200$ c.1. & $3002(11)$ & $2537(5)$ & $393(6)$ & $266.2(5)$ & $-39(7)$ & $-110(29)$ \\
1.1. & $2962(11)$ & $2497(5)$ & & $228.4(5)$ & $-39(7)$ & \\
$\mathrm{D} 200$ c.1. & $3226(13)$ & $2526(5)$ & $600(8)$ & $270.3(6)$ & $-57(12)$ & $-120(36)$ \\
1.1. & $3185(14)$ & $2485(5)$ & & $232.5(5)$ & $-57(12)$ & \\
E250 c.1. & $3552(36)$ & $2594(12)$ & $826(21)$ & $271.8(40)$ & $-164(50)$ & $-301(120)$ \\
1.1. & $3511(36)$ & $2553(12)$ & & $233.7(34)$ & $-171(51)$ & \\
\hline
\end{tabular}




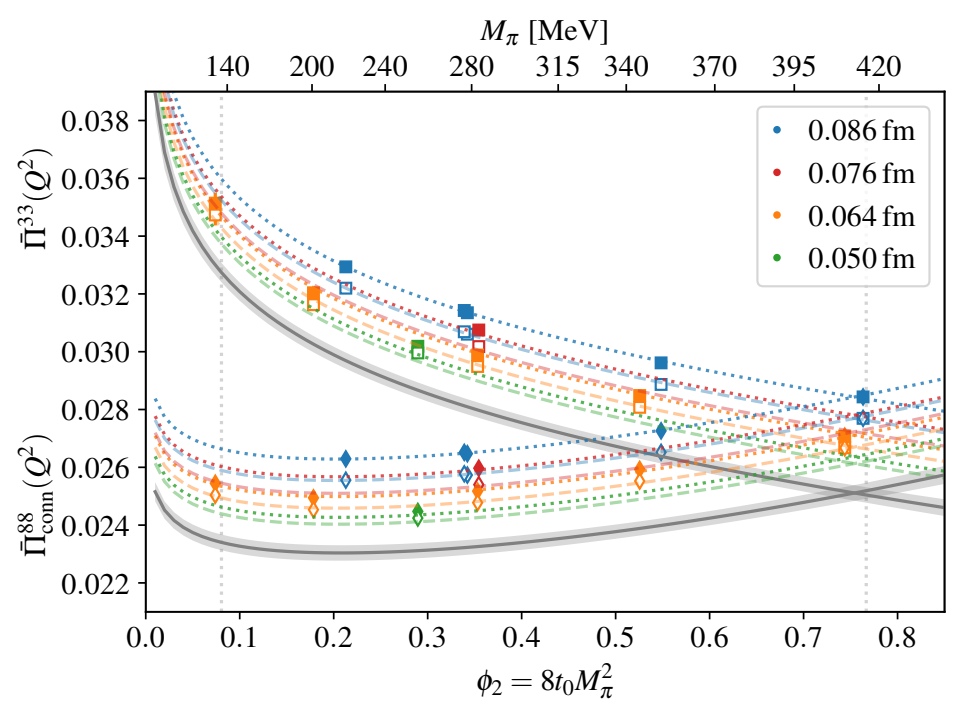

Figure 3: Combined extrapolation of $\bar{\Pi}^{33}\left(Q^{2}\right)$ and $\bar{\Pi}_{\text {conn }}^{88}\left(Q^{2}\right)$ at $Q^{2}=1 \mathrm{GeV}^{2}$ to the physical point. Filled symbols denote the conserved, local discretization, while open symbols denote the local, local one.

extrapolate our results to the physical point. In these proceedings, we consider only the extrapolation of the light and strange connected contributions, which is obtained with a combined fit of the two discretizations of $\bar{\Pi}^{33}\left(Q^{2}\right)$ and $\bar{\Pi}_{\text {conn }}^{88}\left(Q^{2}\right)$. At a given fixed $Q^{2}$, each HVP function contribution in the combined fit is modelled with

$$
\begin{aligned}
\bar{\Pi}\left(a^{2} / t_{0}, \phi_{2}, \phi_{4}\right) & =\bar{\Pi}^{\mathrm{sym}}+\delta_{2} a^{2} / t_{0}+\delta_{3}\left(a^{2} / t_{0}\right)^{3 / 2}+\beta_{21} a^{2}\left(\phi_{2}-\phi_{2}^{\mathrm{sym}}\right) / t_{0} \\
& +\gamma_{1}\left(\phi_{2}-\phi_{2}^{\mathrm{sym}}\right)+\gamma_{2}\left(\log \phi_{2}-\log \phi_{2}^{\mathrm{sym}}\right)+\gamma_{4}\left(\phi_{2}-\phi_{2}^{\mathrm{sym}}\right)^{2}+\eta_{1}\left(\phi_{4}-\phi_{4}^{\mathrm{sym}}\right),
\end{aligned}
$$

where $\phi_{2}=8 t_{0}^{\mathrm{sym}} M_{\pi}^{2}$ and $\phi_{4}=8 t_{0}^{\mathrm{sym}}\left(M_{K}^{2}+M_{\pi}^{2} / 2\right)$ are proxies for the $M_{\pi}$ and $M_{K}$ dependence. For the continuum limit extrapolation, $a^{2}$ and $a^{3}$ terms are included with different coefficients for the two different discretizations. To fit the dependence on the meson masses, we considered different models. In the fit shown in Figure 3, the $\bar{\Pi}^{33}\left(Q^{2}\right)$ interpolation includes the $\gamma_{1}^{33}$ term $\sim M_{\pi}^{2}$ and the $\gamma_{2}^{33}$ term $\sim \log M_{\pi}^{2}$, while the $\bar{\Pi}_{\text {conn }}^{88}\left(Q^{2}\right)$ interpolation includes an independent $\gamma_{1}^{88}$ term and a

Table 3: Results extrapolated to the physical point for the different connected contributions to $\bar{\Pi}$ for a range of $Q^{2}$ values. Only the connected contribution is included in $\Delta \alpha_{\text {had }}$ and $\Delta_{\text {had }} \sin ^{2} \theta_{\mathrm{W}}$.

\begin{tabular}{cccccc}
\hline$Q^{2}\left[\mathrm{GeV}^{2}\right]$ & $\bar{\Pi}^{33}$ & \multicolumn{1}{c}{$\bar{\Pi}_{\text {conn }}^{88}$} & \multicolumn{1}{c}{$\bar{\Pi}_{\text {conn }}^{08}$} & \multicolumn{1}{c}{$\Delta \alpha_{\text {had }}$} & $\Delta_{\text {had }} \sin ^{2} \theta_{\mathrm{W}}$ \\
\hline 0.1 & $0.00764(16)$ & $0.00456(8)$ & $0.00267(8)$ & $0.000841(16)$ & $-0.000821(15)$ \\
0.4 & $0.02068(26)$ & $0.01354(19)$ & $0.00619(12)$ & $0.002310(29)$ & $-0.002300(29)$ \\
1.0 & $0.03274(34)$ & $0.02340(27)$ & $0.00809(13)$ & $0.003718(38)$ & $-0.003770(40)$ \\
2.0 & $0.04242(38)$ & $0.03226(33)$ & $0.00880(13)$ & $0.004876(45)$ & $-0.005012(47)$ \\
3.0 & $0.04805(41)$ & $0.03768(36)$ & $0.00898(13)$ & $0.005558(48)$ & $-0.005752(50)$ \\
4.0 & $0.05202(42)$ & $0.04157(38)$ & $0.00904(13)$ & $0.006041(50)$ & $-0.006278(53)$ \\
5.0 & $0.05508(43)$ & $0.04461(39)$ & $0.00907(13)$ & $0.006415(51)$ & $-0.006687(54)$ \\
\hline
\end{tabular}


$\gamma_{4}^{88}$ term $\sim M_{\pi}^{4}$, while we constrain $\gamma_{2}^{88}=\gamma_{2}^{33} / 3$. The latter term is included to match the diverging behaviour of $\bar{\Pi}^{33}\left(Q^{2}\right)$ with $M_{\pi} \rightarrow 0$, that is present in $\bar{\Pi}_{\text {conn }}^{88}\left(Q^{2}\right)$, with a factor of one third, due to the missing disconnected contribution [26].

The combined fit includes the uncertainties in the determination of the pion and kaon masses, including the correlation with the HVP function determination, that are obtained from a dedicated computation. For every ensemble $l$, we build a residue vector

$$
v_{l}=\left(\begin{array}{c}
\phi_{2}^{l} \\
\bar{\Pi}_{\mathrm{cl}}^{33}\left(a^{2} / t_{0}^{\mathrm{sym}}, \phi_{2}^{l}, \phi_{4}^{l}\right) \\
\bar{\Pi}_{11}^{33}\left(a^{2} / t_{0}^{\mathrm{sym}}, \phi_{2}^{l}, \phi_{4}^{l}\right) \\
\phi_{4}^{l} \\
\bar{\Pi}_{\mathrm{cl}}^{88}\left(a^{2} / t_{0}^{\mathrm{sym}}, \phi_{2}^{l}, \phi_{4}^{l}\right) \\
\bar{\Pi}_{11}^{88}\left(a^{2} / t_{0}^{\mathrm{sym}}, \phi_{2}^{l}, \phi_{4}^{l}\right)
\end{array}\right)-\left(\begin{array}{c}
8 t_{0}^{\mathrm{sym}} M_{\pi}^{2} \\
\bar{\Pi}_{\mathrm{cl}}^{33} \\
\bar{\Pi}_{11}^{33} \\
8 t_{0}^{\mathrm{sym}}\left(M_{K}^{2}+M_{\pi}^{2} / 2\right) \\
\bar{\Pi}_{\mathrm{cl}}^{88} \\
\bar{\Pi}_{11}^{88}
\end{array}\right)_{\text {data }}
$$

and we minimize the $\chi^{2}=\sum_{l \in\{\text { ensembles }\}} v_{l}^{\top} C_{l}^{-1} v_{l}$, with the necessary adaptations for SU(3)symmetric ensembles where $\bar{\Pi}^{33}$ and $\bar{\Pi}^{88}$, and $M_{\pi}$ and $M_{K}$, are not independent.

This choice leads to an acceptable fit with an additional cut on the physical lattice volume that excludes lattices with $L<2.5 \mathrm{fm}$. At $Q^{2}=1 \mathrm{GeV}^{2}$, we have $\chi^{2} /$ dof $=52.13 / 38=1.37$, which corresponds to a $p$-values of 0.0631 , and parameters

$$
\begin{gathered}
\bar{\Pi}^{\mathrm{sym}}=0.02516(29), \quad \phi_{2}^{\mathrm{sym}}=2 \phi_{4}^{\mathrm{sym}} / 3=0.754(5), \quad \eta_{1}=-0.011(4), \\
\delta_{2}^{\mathrm{cl}}=0.0126(31), \quad \delta_{2}^{11}=0.0106(31), \quad \delta_{3}^{\mathrm{cl}}=-0.005(4), \quad \delta_{3}^{11}=-0.006(4), \quad \beta_{21}=0.0004(23), \\
\gamma_{1}^{33}=-0.0017(9), \quad \gamma_{1}^{88}=0.00363(33), \quad \gamma_{2}^{33}=\gamma_{2}^{88} / 3=-0.00291(28), \quad \gamma_{4}^{88}=0.0026(5) .
\end{gathered}
$$

The extrapolation to $a=0$ and to physical $\pi^{0}$ and $K^{0}$ masses gives $\bar{\Pi}^{33}=0.03274(34)$ and $\bar{\Pi}_{\text {conn }}^{88}=$ $0.02340(27)$. Moreover, $\bar{\Pi}_{\text {conn }}^{08}=\sqrt{3}\left(\bar{\Pi}^{33}-\bar{\Pi}_{\text {conn }}^{88}\right) / 2=0.00809$ (13). Applying Eqs (1.2) and (2.1) allows us to compute the leading hadronic connected contribution from three-flavour QCD to the running of $\alpha$ and $\sin ^{2} \theta_{\mathrm{W}}$ at $Q^{2}=1 \mathrm{GeV}^{2}$

$$
\Delta \alpha_{\text {had }}\left(-1 \mathrm{GeV}^{2}\right)=0.003718(38), \quad \Delta_{\mathrm{had}} \sin ^{2} \theta_{\mathrm{W}}\left(-1 \mathrm{GeV}^{2}\right)=-0.003770(40) .
$$

\subsection{The running with energy}

Performing the fit described in Section 5.1 for different values of $Q^{2}$ results in a mild dependence of the $\chi^{2}$ on $Q^{2}$ up to around $2 \mathrm{GeV}^{2}$. At higher $Q^{2}$ discretization effects become the dominant systematics and the quality of the fit, using the current model, starts to deteriorate. Results extrapolated to the physical point for a number of $Q^{2}$ values are given in Table 3, and in Figure 4 we plot the HVP contribution to the running of $\alpha$ and $\sin ^{2} \theta_{\mathrm{W}}$. In both plots, we compare our preliminary result to the corresponding contribution from the percent-level lattice determination of Ref. [5], given in the supplemental material for five $Q^{2}$ values. The agreement is good, with a small tension only at the smallest $Q^{2}=1 \mathrm{GeV}^{2}$.

\section{Conclusions and outlook}

We computed on the lattice the leading hadronic contribution to the running of the electromagnetic coupling $\alpha$ and of the electroweak mixing angle $\theta_{\mathrm{W}}$. After extrapolating to the physical 

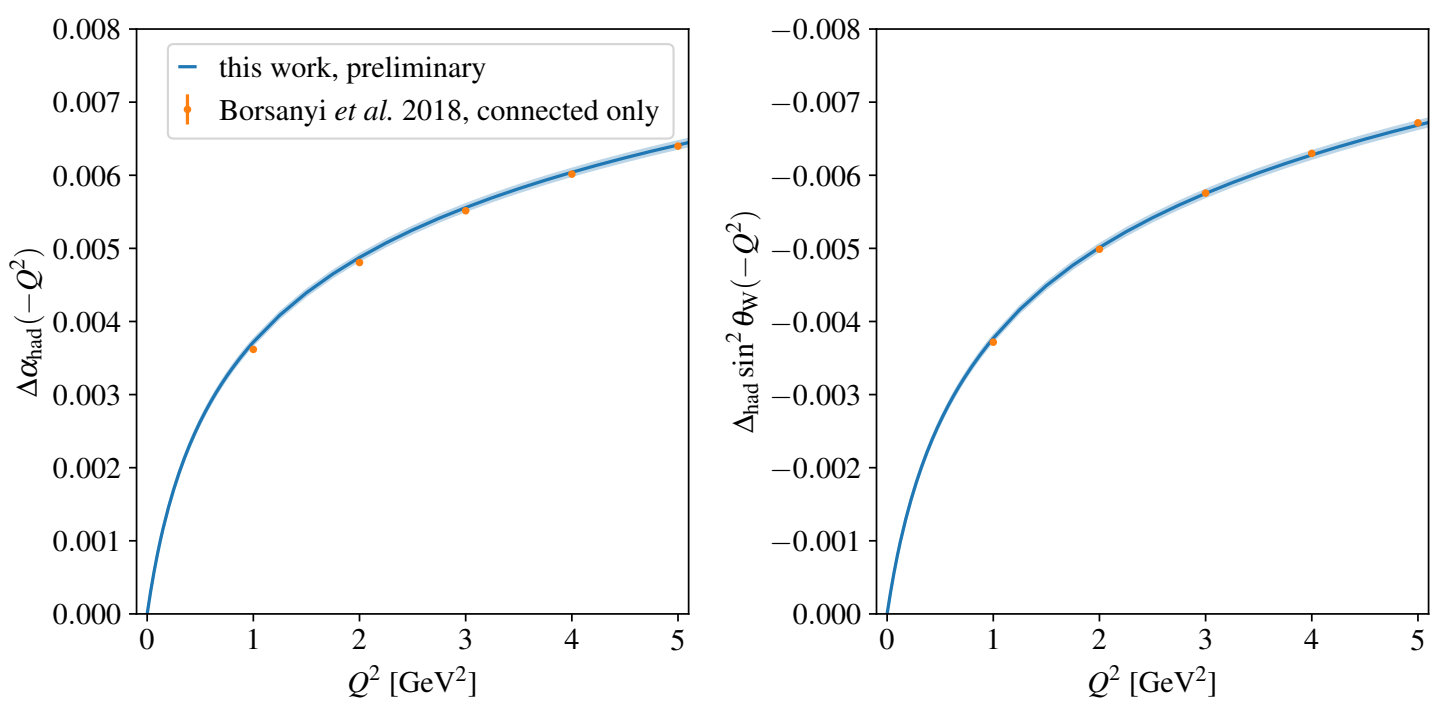

Figure 4: Leading hadronic (connected) contribution from three-flavour QCD to the running of the electromagnetic coupling $\alpha$ (left) and the electroweak mixing angle $\sin ^{2} \theta_{\mathrm{W}}$ (right) as a function of the space-like momentum transfer $Q^{2}$. The blue line (with error band) is the extrapolation of our lattice data, while the orange points are constructed from the HVP values from Table S3 of the supplemental material of Ref. [5].

point, the statistical error on the connected contribution amounts to $\approx 4 \times 10^{-5}$ which, for both quantities, is $1 \%$ of the contribution at $Q^{2}=1 \mathrm{GeV}^{2}$. We also presented results for the disconnected contribution, not yet extrapolated at the physical point, which show that the statistical error is also around $1 \%$ of the total contribution. We included finite-volume corrections, that are instrumental for obtaining a reliable physical-point extrapolation. The precision of our results is comparable to the phenomenological estimate, which is around $0.7 \%$ and $1 \%$ for the two quantities respectively [40]. In particular, flavour contributions are naturally singled out on the lattice. Thus, our results can be combined with the phenomenological analysis of the electroweak mixing angle to reduce its flavour separation systematics.

We are currently working on increasing the statistics of disconnected loops at physical meson masses. Subsequently, we will extrapolate the disconnected and the quenched charm contribution to the physical point. Moreover, a full assessment of systematic errors is missing from the results in Table 3. We plan to assess the systematics introduced by the extrapolation by varying the choice of the fit function, and, in the future, adding a new ensemble at the fine lattice spacing $a \approx 0.050 \mathrm{fm}$ with a lighter pion mass of $M_{\pi} \approx 175 \mathrm{MeV}$. We plan, as described in Section 4.4, to improve the treatment of the long-time tail of the lighter ensembles with the bounding method. The scale setting introduces an error that can be estimated as explained in Section B.2 of Ref. [21]. Also, a small systematics from the mistuning of the charm hopping parameter needs to be added. Finally, to compare with the physical world, isospin-breaking effects from non-degenerate $u$ and $d$ quark masses and QED are to be included [41, 42].

Acknowledgements: We thank Jens Erler, Fred Jegerlehner and Daniel Mohler for valuable discussions. Calculations for this project have been performed on the HPC clusters "clover" and "himster2" at Helmoltz-Institut Mainz and "Mogon II" at JGU Mainz, on the BG/Q system "JUQUEEN" at Jülich Supercomputing Center (JSC), and on "Hazel Hen" at 
Höchstleistungsrechenzentrum Stuttgart (HLRS). The authors gratefully acknowledge the support of the Gauss Centre for Supercomputing and the John von Neumann-Institut für Computing (NIC) for project HMZ21 at JSC and project GCS-HQCD at HLRS. Our programs use the deflated SAP + GCR solver from the OPENQCD package, as well as the QDP++ library. We are grateful to our colleagues in the CLS initiative for sharing ensembles.

\section{References}

[1] Particle Data Group collaboration, Review of Particle Physics, Phys. Rev. D 98 (2018) 030001.

[2] F. Jegerlehner, Electroweak effective couplings for future precision experiments, Nuovo Cim. C 034S1 (2011) 31 [1107.4683].

[3] F. Burger, K. Jansen, M. Petschlies and G. Pientka, Leading hadronic contributions to the running of the electroweak coupling constants from lattice QCD, JHEP 1511 (2015) 215 [1505. 03283].

[4] A. Francis, V. Gülpers, G. Herdoíza, H. Horch, B. Jäger, H. B. Meyer et al., Study of the hadronic contributions to the running of the QED coupling and the weak mixing angle, PoS LATTICE2015 (2015) 110 [1511.04751].

[5] BudAPEST-MARSEILle-WuPPERTAL collaboration, Hadronic vacuum polarization contribution to the anomalous magnetic moments of leptons from first principles, Phys. Rev. Lett. 121 (2018) 022002 [1711.04980].

[6] H. B. Meyer and H. Wittig, Lattice QCD and the anomalous magnetic moment of the muon, Prog. Part. Nucl. Phys. 104 (2019) 46 [1807.09370].

[7] M. Passera, W. J. Marciano and A. Sirlin, The muon $g-2$ and the bounds on the Higgs boson mass, Phys. Rev. D 78 (2008) 013009 [0804.1142].

[8] G. Abbiendi, C. M. C. Calame, U. Marconi, C. Matteuzzi, G. Montagna, O. Nicrosini et al., Measuring the leading hadronic contribution to the muon $g-2$ via $\mu$ e scattering, Eur. Phys. J. C 77 (2017) 139 [1609.08987].

[9] G. Abbiendi, Letter of Intent: the MUonE project, Tech. Rep. CERN-SPSC-2019-026. SPSC-I-252, CERN, Geneva, June, 2019.

[10] B. E. Lautrup, A. Peterman and E. de Rafael, Recent developments in the comparison between theory and experiments in quantum electrodynamics, Phys. Rept. 3 (1972) 193.

[11] M. Krstić Marinković, "Leading hadronic contribution to muon $g-2$ from lattice QCD and the MUonE experiment." talk at The 36th Annual International Symposium on Lattice Field Theory, 2018.

[12] A. Ferroglia, G. Ossola and A. Sirlin, The electroweak form factor $\hat{\kappa}\left(q^{2}\right)$ and the running of $\sin ^{2} \hat{\theta}_{\mathrm{W}}$, Eur. Phys. J. C 34 (2004) 165 [hep-ph/0307200].

[13] K. S. Kumar, S. Mantry, W. J. Marciano and P. A. Souder, Low energy measurements of the weak mixing angle, Ann. Rev. Nucl. Part. Sci. 63 (2013) 237 [1302 . 6263].

[14] MOLLER collaboration, The MOLLER experiment: An ultra-precise measurement of the weak mixing angle using Moller scattering, 1411.4088.

[15] D. Becker, R. Bucoveanu, C. Grzesik, K. Imai, R. Kempf, M. Molitor et al., The P2 experiment, Eur. Phys. J. A 54 (2018) [1802.04759].

[16] J. Erler and R. Ferro-Hernández, Weak mixing angle in the Thomson limit, JHEP 1803 (2018) 196 [1712.09146].

[17] F. Jegerlehner, Hadronic contributions to electroweak parameter shifts, Z. Phys. C Part. Fields 32 (1986) 195.

[18] V. Gülpers, H. Meyer, G. von Hippel and H. Wittig, The leading hadronic contribution to $\gamma-Z$ mixing, PoS LATTICE2015 (2016) 263.

[19] M. Cè, A. Gérardin, K. Ottnad and H. B. Meyer, The leading hadronic contribution to the running of the weinberg angle using covariant coordinate-space methods, PoS LATTICE2018 (2018) 137 [1811.08669]. 
[20] D. Bernecker and H. B. Meyer, Vector correlators in lattice QCD: Methods and applications, Eur. Phys. J. A 47 (2011) 148 [1107.4388].

[21] M. Della Morte, A. Francis, V. Gülpers, G. Herdoíza, G. von Hippel, H. Horch et al., The hadronic vacuum polarization contribution to the muon $g-2$ from lattice QCD, JHEP 1710 (2017) 020 [1705.01775].

[22] A. Francis, B. Jaeger, H. B. Meyer and H. Wittig, New representation of the Adler function for lattice QCD, Phys. Rev. D 88 (2013) 054502 [1306.2532].

[23] M. Bruno, D. Djukanovic, G. P. Engel, A. Francis, G. Herdoiza, H. Horch et al., Simulation of QCD with $N_{\mathrm{f}}=2+1$ flavors of non-perturbatively improved Wilson fermions, JHEP 1502 (2015) 043 [1411.3982].

[24] M. Lüscher, Properties and uses of the Wilson flow in lattice QCD, JHEP 1008 (2010) 071 [1006.4518].

[25] M. Bruno, T. Korzec and S. Schaefer, Setting the scale for the CLS $2+1$ flavor ensembles, Phys. Rev. D 95 (2017) 074504 [1608.08900].

[26] A. Gérardin, M. Cè, G. von Hippel, B. Hörz, H. B. Meyer, D. Mohler et al., Leading hadronic contribution to $(g-2)_{\mu}$ from lattice $Q C D$ with $N_{\mathrm{f}}=2+1$ flavors of $\mathrm{O}($ a) improved Wilson quarks, Phys. Rev. D 100 (2019) 014510 [1904.03120].

[27] T. Bhattacharya, R. Gupta, W. Lee, S. R. Sharpe and J. M. S. Wu, Improved bilinears in lattice QCD with nondegenerate quarks, Phys. Rev. D 73 (2006) 034504 [hep-lat/ 0511014 ].

[28] A. Gerardin, T. Harris and H. B. Meyer, Non-perturbative renormalization and $\mathrm{O}($ a)-improvement of the non-singlet vector current with $N_{\mathrm{f}}=2+1$ Wilson fermions and tree-level Symanzik improved gauge action, Phys. Rev. D 99 (2018) [1811.08209].

[29] ALPHA collaboration, Monte Carlo errors with less errors, Comput. Phys. Commun. 156 (2004) 143 [hep-lat/0306017].

[30] B. De Palma, M. Erba, L. Mantovani and N. Mosco, A Python program for the implementation of the Г-method for Monte Carlo simulations, Comput. Phys. Commun. 234 (2019) 294 [1703 . 02766].

[31] M. Lüscher, Signatures of unstable particles in finite volume, Nucl. Phys. B 364 (1991) 237.

[32] L. Lellouch and M. Lüscher, Weak transition matrix elements from finite-volume correlation functions, Commun. Math. Phys. 219 (2001) 31 [hep-lat/ 0003023 ].

[33] H. B. Meyer, Lattice QCD and the Timelike Pion Form Factor, Phys. Rev. Lett. 107 (2011) 072002 [1105.1892].

[34] G. J. Gounaris and J. J. Sakurai, Finite-width corrections to the vector-meson-dominance prediction for $\rho \rightarrow e+e-$, Phys. Rev. Lett. 21 (1968) 244.

[35] C. Andersen, J. Bulava, B. Hörz and C. Morningstar, The $I=1$ pion-pion scattering amplitude and timelike pion form factor from $N_{\mathrm{f}}=2+1$ lattice QCD, Nucl. Phys. B 939 (2019) 145 [1808.05007].

[36] F. Erben, J. R. Green, D. Mohler and H. Wittig, Rho resonance, timelike pion form factor, and implications for lattice studies of the hadronic vacuum polarisation, 1910.01083.

[37] C. Aubin, T. Blum, P. Chau, M. Golterman, S. Peris and C. Tu, Finite-volume effects in the muon anomalous magnetic moment on the lattice, Phys. Rev. D 93 (2016) 054508 [1512. 07555 ].

[38] M. T. Hansen and A. Patella, Finite-volume effects in $(g-2)_{\mu}^{H V P, L O}$, Phys. Rev. Lett. 123 (2019) [1904.10010].

[39] C. Lehner, "The hadronic vacuum polarization contributionto the muon anomalous magnetic moment." talk at RBRC Workshop on Lattice Gauge Theories.

[40] F. Jegerlehner, Variations on photon vacuum polarization, 1711.06089.

[41] A. Risch and H. Wittig, Towards leading isospin breaking effects in mesonic masses with open boundaries, PoS LATTICE2018 (2019) 059 [1811. 00895 ].

[42] A. Risch and H. Wittig, Leading isospin breaking effects in the hadronic vacuum polarisation with open boundaries, PoS LATTICE2019 (2019) 296 [1911. 04230 ]. 